\title{
Design and Simulation of
}

\section{an Electromagnetic Shock Absorber}

\author{
Jae-Sung Bae, Jai-Hyuk Hwang, Jung-Sun Park, Mi-Seon Yi
}

\begin{abstract}
The present study proposed an electromagnetic shock-absorber (EMSA) consisting of a copper and steel combined tube, a piston, permanent magnets, and a steel ring. A magnet fixed on the piston moves through the tube when driven by an external shock. This shock energy is partially dissipated by an eddy current damping force and a friction force generated from the relative motion of the magnet and the tube. A part of the energy is stored in a magnetic spring consisting of two magnets. To predict the performance of the proposed EMSA these damping and magnetic forces are simulated and approximated by using an electromagnetic finite element program. The present simulation results are verified by the experimental results.
\end{abstract}

Keywords - Electromagnetic Shock Absorber (EMSA), Eddy Current Damping (ECD), friction, attractive and propulsive magnetic force

\section{Introduction}

Eddy currents are generated when a moving conducting material intersects a stationary magnetic field, or vice-versa. These eddy currents induce their own magnetic field with the opposite polarity of the applied field that causes a resistive force. These currents dissipate due to the electrical resistance and this force will eventually disappear. Hence, the vibrating energy will be dissipated. Since the resistive force induced by eddy currents is proportional to the relative velocity, this eddy current damping can be allowed to function as a form of viscous damping.

Bae et al. [1] developed a theoretical model of an ECD constructed by Kwak et al. [2]. Using this theoretical model, they investigated the damping characteristics of an ECD and simulated the vibration suppression of a cantilever beam with Kwak's ECD. Sodano et al. [3-5] proposed a new concept using the eddy currents induced in a conductive plate to suppress the vibration of a cantilevered beam. Bae et al.[6] proposed the concept of magnetically tuned-mass-damper (mTMD) to improve the damping performance of a conventional TMD by using an eddy current damping (ECD). They showed that their method could significantly increase the damping effect of the TMD by simulations and experiments. Bae et al. [7] proposed and exploited an effective method to

\footnotetext{
J.S. Bae, Jai-Hyuk Hwang, Jung-Sun Park

Korea Aerospace University

Republic of Korea

M.S. Yi

Agency for Defense Development

Republic of Korea
}

suppress the vibration of a large and heavy beam structure with a minimum increase in mass or volume of material. They showed that their method is simple but effective in suppressing the vibration of a large beam structure without a substantial weight increase.

The objective of the present study is to design and simulate new electromagnetic shock absorber (EMSA) for a landing structure of a spaceship. First the present study proposed new concept of an EMSA consisting of a moving piston, a cooper/steel combined tube, and permanent magnets. Second the present study analyzes a shock absorbing mechanism by each element, performs the electromagnetic analysis for each element, and presents the numerical model of the EMSA.

\section{Electromagnetic Shock Absorber}

\section{A. Mechanism of Shock Absorption}

Figure 1 shows the three-step shock absorbing mechanism of the proposed EMSA. (1) The first step is : When EMSA was shocked the piston and the magnet starts to move downward but are opposed to moving by attractive magnetic force between the magnet and the steel end of the tube.

(2) The second step is : When the magnet is moving there exist two kinds of forces. One is eddy current damping force between the moving magnet and the stationary copper tube. The other is a friction force between the magnet and the tube.

(3) The last step is : When the magnet approaches to the other end of the tube it is opposed to move by repulsive magnetic force between the moving magnet and the magnet fixed at the lower end of the tube.

In these three processes the second step absorbs the most of impulse energy.

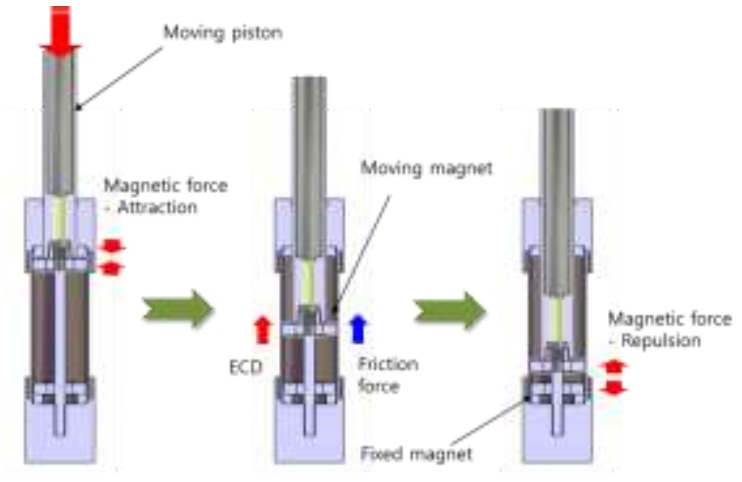

Figure 1 The proposed mechanism of shock absorption 


\section{B. Predictions of magnetic, ECD, and friction forces}

In this section the electromagnetic analyses are performed to predict attractive magnetic force between the magnet and the steel ring, ECD force, friction force, and repulsive magnetic force between the magnets. The present study uses a commercial program of Ansoft's MAXWELL which is a finite element program to solve an electromagnetics. Figure 2(a) shows the simulation model to calculate an attractive magnetic force between the magnet and the steel ring. Since the magnet and the steel ring are axis-symmetric they are modeled in the $r$ (radius) $-\mathrm{z}$ plane to save computational costs. Figure 2(b) shows the attractive magnetic force between the magnet and the steel ring. This is strong where the magnet places at the vicinity of the tube end and the maximum value is approximately $120 \mathrm{~N}$.

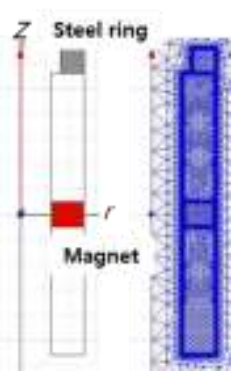

(a)

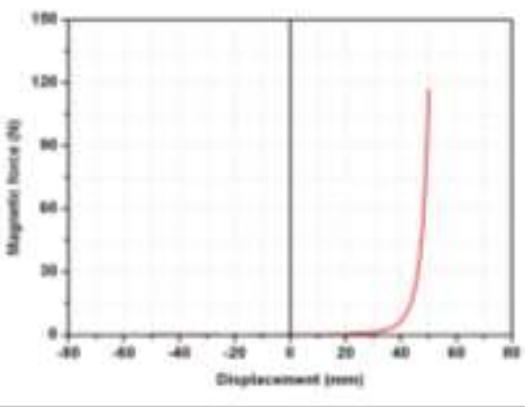

(b)
Figure 2 Attractive magnetic force between magnet and steel ring. (a) simulation model and FEM grid; (b) Force-displace plot

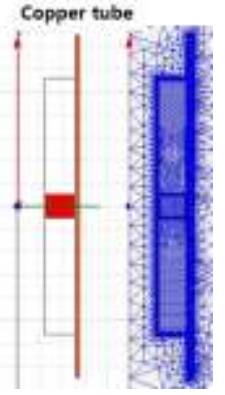

(a)

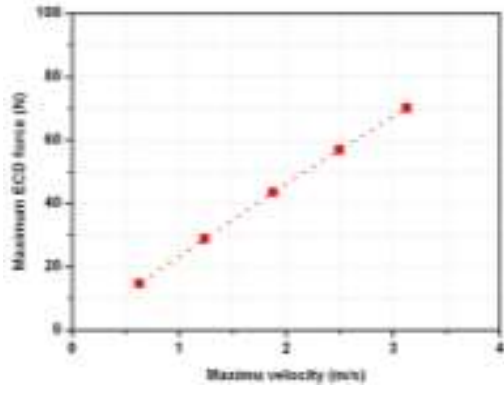

(b)
Figure 3 ECD force between the magnet and the copper tube. (a) simulation model and FEM grid; (b) maximum ECD force vs. maximu velocity.

Figure 3 shows the simulation model to calculate ECD force between the magnet and the copper tube and maximum ECD force - maximum velocity plot. Figure 4 shows the force-displacement and force-velocity plots of ECD force for various excitation frequencies. Same as the previous case ECD force is also modeled in the r-z plane.

Figure 5 shows the simulation model and its FEM grid to calculate normal force which is a magnetic force between the magnet and the 90 degree steel tube. This normal force is used to calculate the friction force between the magnet and the copper tube. When the steel is circular and the magnet in in equilibrium in the tube the normal force is zero. Physically since there is a gap between the magnet and tube the magnet is not in equilibrium and there exist normal force. But this force is less than that of the 90 degree steel tube. The magnetic force between the magnet and the steel tube is $102.6 \mathrm{~N}$ and the friction force is about $41 \mathrm{~N}$ when a kinetic friction coefficient is assumed to be 0.4 .

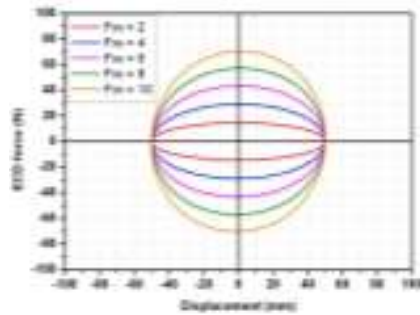

(a)

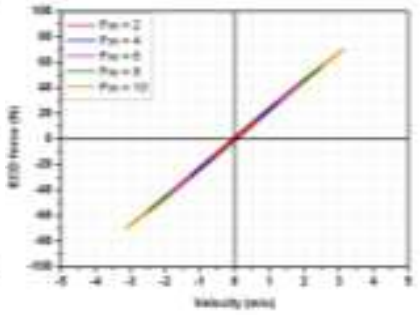

(b)
Figure 4 ECD force. (a) force vs. displacement; (b) force vs. velocity.

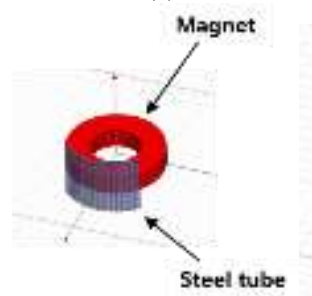

(a)

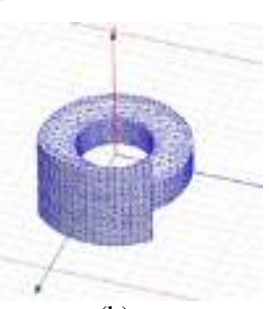

(b)
Figure 5 Friction force. (a) simulation model; (b) FEM grid.

Figure 6 (a) shows the simulation model and its FEM grid to calculate the repulsive magnetic force between the moving and fixed magnets. Same as Figures 2(a) and 3(a) this is also model in the r-z plane. Figure 6(b) shows the forcedisplacement plot of this repulsive magnetic force. This force plays a role of spring force and the relation is quadratic or cubic.
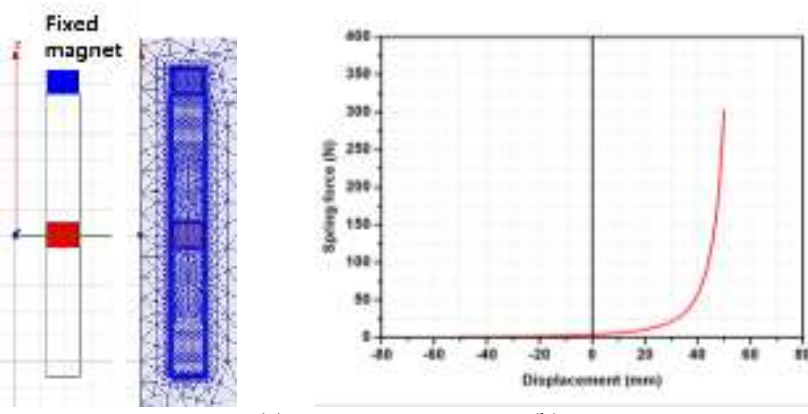

(a)

(b)

Figure 6 Repulsive magnetic force (a) simulation model and FEM grid; (b) force-displacement plot.

\section{Verifications with Experiments}

The present study verifies the simulation results of four kinds of forces with experiments. Figure. 7 shows the experimental setup to measure attractive/repulsive magnetic, $\mathrm{ECD}$, and friction forces. The piston is excited by $\mathrm{AC}$ motor and the excitation is harmonic. The displacement and force are measured by LVDT and load cell, respectively. The maximum 
amplitude of excitation is about $50 \mathrm{~mm}$. Due to the limitation of actuation force the maximum excitation frequency is about $8.5 \mathrm{~Hz}$. The simulations of four kinds of forces are verified by this experimental setup as shown in Figure 7. Using the simulation models of four kinds of forces the performance of the proposed EMSA can be predicted. Figure 8 shows the performance prediction of the EMSA when the piston is assumed to be in uniform motion. When the piston starts to move the force becomes large due to presence of attractive magnetic force. When the piston is between $20 \mathrm{~mm}$ and 80 $\mathrm{mm}$ the force is almost constant due to ECD force and friction force. Friction force is theoretically constant and ECD force is also constant when the piston is in uniform motion. As the piston goes to the end the force increases exponentially due to repulsive magnetic force.

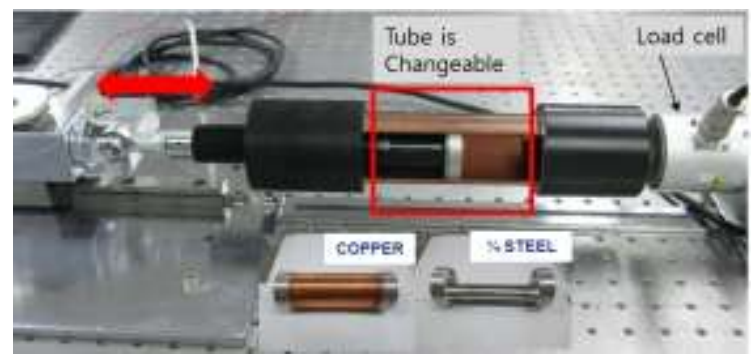

Figure 7 Experimental setup

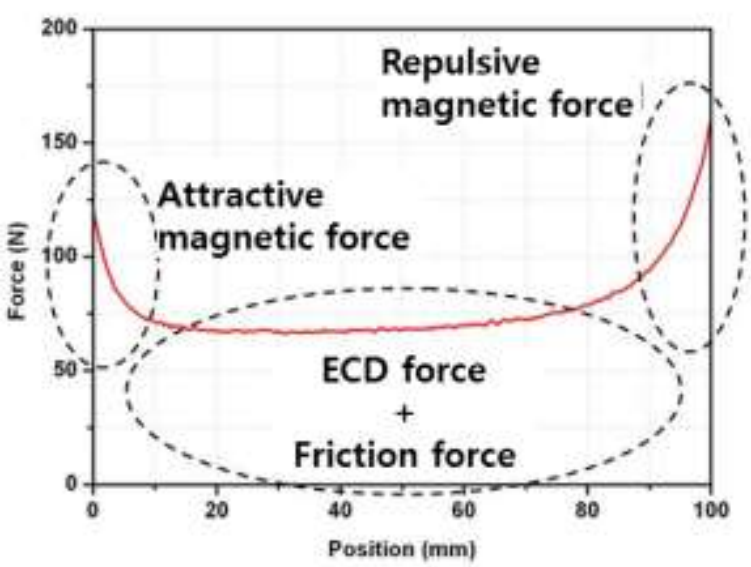

Figure 8 Predicted performance of EMSA in uniform motion

\section{Conclusions}

A conventional shock absorber with air and fluid may not be applicable to space structures because of leakage. An electromagnetic damper such as an eddy current damper is useful in space because it does not have any problems with leakage. The present study designed and simulated a new electromagnetic shock absorber for a landing structure of a spaceship. The proposed EMSA consists of a copper and steel combined tube, a piston, moving and fixed magnets, and a steel ring. The shock absorbing process includes dissipating energy by eddy current damping and friction forces between the tube and moving magnet and restoring energy in the magnetic spring of two magnets.

\section{Acknowledgment}

This work was supported by Global Surveilance Research Center(GSRC) program funded by the Defense Acquistion Program Administration(DAPA) and Agency for Defense Development. And this work was partially supported by ADD (ADD-13-01-08-24).

\section{References}

[1] J.S. Bae, M.K. Kwak, D.J. Inman, Vibration Suppression of Cantilever Beam Using Eddy Current Damper. Journal of Sound and Vibration, 284(2005) 805-824.

[2] M. K. Kwak, M. I. Lee, S. Heo, Vibration Suppression Using Eddy Current Damper. Korean Society for Noise and Vibration Engineering 233(2003) 441-453.

[3] H.A. Sodano, J.S. Bae, D.J. Inman, W.K. Belvin, Concept and Model of Eddy Current Damper for Vibration Suppression of a Beam. Journal of Sound and Vibration 288(2005) 1177-1196.

[4] H.A. Sodano, J.S. Bae, D.J. Inman, W.K. Belvin, Modeling and Application of Eddy Current Damper for Suppression of Membrane Vibration. AIAA Journal, 44(2006) 541-548.

[5] H.A. Sodano, J.S. Bae, D.J. Inman, W.K. Belvin, Improved Concept and Model of Eddy Current Damper. Journal of Vibration and Acoustics, 128(2006) 294-302.

[6] J.S. Bae, J.H. Hwang, J.H. Roh, J.H. Kim, M.S. Yi, J.H. Lim, Vibration Suppression of a Cantilever Beam using Magnetically Tuned-MassDamper. Journal of Sound and Vibration 331 (2012) 5669-5684.

[7] J.S. Bae, J.H. Hwang, D.G. Kwag, D.J. Inman, Vibration Suppression of a Large Beam Structure Using Tuned-Mass-Damper and Eddy Current Damping. Shock and Vibration, Volume 2014, Article ID 893914.

About Author (s):

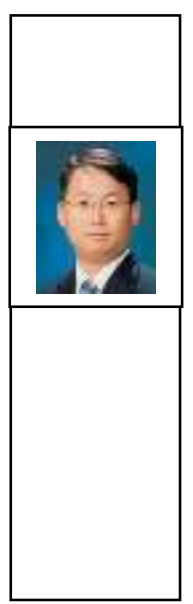

Jae-Sung Bae received his B.S. degree in Aeronautical and Mechanical Engineering from Korea Aerospace University in 1996 and his M.S. degree in Aerospace Engineering from KAIST in 1998. He then received his Ph.D. in Aerospace Engineering from KAIST in 2002. Dr. Bae is currently an Associate Professor at the School of Aerospace and Mechanical Engineering at Korea Aerospace

University in Goyang-City, Korea. His main research interests include aeroelasticity, vibration and control, shock absorber, and UAV system 